\title{
A THEOREM IN THE THEORY OF SUMMABILITY
}

BY J. D. HILL

1. Introduction. Knopp* has developed a general integral form for linear methods of summability which includes as special cases all such methods which to date have found any considerable application. He establishes sufficient conditions for the regularity of his method but does not completely treat the question of their necessity. It may be of interest to observe that if Knopp's integral is interpreted in the sense of Lebesgue one may readily construct an example of his method which is regular in the class of bounded, measurable functions, but which does not satisfy condition (b) $\dagger$ of his regularity theorem. In conformity with Knopp's notation, let the curves $\mathfrak{S}_{x}, \mathfrak{S}_{y}$ be taken as the real axes $0 \leqq x<\infty, 0 \leqq y<\infty$, respectively. Denote by $(\mathfrak{U})$ the class of all complex functions $f(x) \equiv f_{1}(x)+i f_{2}(x)$ defined on $\mathfrak{S}_{x}$ such that $f_{1}(x), f_{2}(x)$ are bounded and measurable on the interval $0 \leqq x \leqq X$ for every $X>0$, and such that $\lim _{x \rightarrow \infty} f(x) \equiv L_{f}$ exists. Finally, let the function $K(x, y)$ be defined as $(-1)^{j}$ for $\quad n-1 \leqq y<n, \quad(n=1,2,3, \cdots), \quad(j-1) / 2^{n} \leqq x<j / 2^{n}$, $\left(j=1,2, \cdots, 2^{n}\right) ;$ as $1 /(y+1)$ for $1 \leqq x \leqq y+1,0 \leqq y<\infty$; and as zero for $0 \leqq y<x-1,1<x<\infty$. Then for every $f(x) \subset(\mathfrak{U})$, $g(y) \equiv \int_{0}^{y+1} K(x, y) f(x) d x$ clearly exists on $\mathfrak{S}_{y}$, and we have

$$
g(y)=\int_{0}^{1} K(x, y) f(x) d x+\int_{1}^{y+1} \frac{1}{y+1} f(x) d x,
$$

where the second integral tends to $L_{f}$ as $y \rightarrow \infty$, since the function $1 /(y+1)$ satisfies the conditions of Knopp's regularity theorem. Moreover, given $\epsilon>0$, there exist step-functions $s_{j}(x)$ such that $\int_{0}^{1}\left|f_{j}(x)-s_{i}(x)\right| d x<\epsilon,(j=1,2)$. Consequently, in view of $|K(x, y)| \leqq 1$, we have

* Knopp, Zur Theorie der Limitierungsverfahren, Mathematische Zeitschrift, vol. 31 (1929-30), pp. 97-127. To save space we assume that the reader is familiar with this paper.

$\dagger$ Loc. cit., p. 101. 


$$
\begin{aligned}
\left|\int_{0}^{1} K(x, y) f(x) d x\right| \leqq & \left|\int_{0}^{1} K(x, y) s_{1}(x) d x\right| \\
& +\left|\int_{0}^{1} K(x, y) s_{2}(x) d x\right|+2 \epsilon
\end{aligned}
$$

$s_{1}(x), s_{2}(x)$ being step-functions, the two integrals on the right tend to zero as $y \rightarrow \infty$. Hence $\lim _{y \rightarrow \infty} g(y)=L_{f}$ and $K(x, y)$ defines a regular method in the class ( $\mathfrak{A})$. On the other hand, $\int_{0}^{1}|K(x, y)| d x$ does not tend to zero as $y \rightarrow \infty$, so that condition (b) in this case is not fulfilled.

This fact leads us to consider a slight modification of Knopp's method, based on the Lebesgue integral, for which it is possible to state conditions necessary as well as sufficient for regularity in the class of functions defined below. Aside from specifying the type of integral used, the modification consists mainly in dispensing with the curves $\mathfrak{C}_{x}, \mathfrak{S}_{y}$ and directing attention to the real and imaginary parts of the complex functions with which we are concerned.

2. K-Method of Summability. Let ( $\mathfrak{B})$ denote the class of all complex functions $f(x) \equiv f_{1}(x)+i f_{2}(x)$ defined on the interval $I \equiv(0 \leqq x<\xi), \xi \leqq \infty$, such that (i) $f_{1}(x), f_{2}(x)$ are measurable on the sub-interval $0 \leqq x \leqq X$ for every $X, 0<X<\xi$, (ii) $|f(x)|$ is essentially bounded on $I$, and (iii) $f(x)$ tends to a limit $L_{f}$ as $x \rightarrow \xi$ on $I$. Let $K(x, y) \equiv K_{1}(x, y)+i K_{2}(x, y)$ be an arbitrary complex function defined on the intervals $I$ and $J \equiv(0 \leqq y<\eta), \eta \leqq \infty$. We shall say that $K(x, y)$ defines a regular method of summability in the class $(\mathfrak{B})$ if for every $f(x) \subset(\mathfrak{B})$ the integral

$$
\begin{aligned}
U_{y}(f) \equiv & \int_{0}^{\xi} K(x, y) f(x) d x \\
\equiv & \lim _{x \rightarrow \xi} \int_{0}^{x}\left[K_{1}(x, y) f_{1}(x)-K_{2}(x, y) f_{2}(x)\right] d x \\
& +i \lim _{x \rightarrow \xi} \int_{0}^{x}\left[K_{1}(x, y) f_{2}(x)+K_{2}(x, y) f_{1}(x)\right] d x
\end{aligned}
$$

exists on $J$, and if $U_{y}(f)$ tends to a limit equal to $L_{f}$ as $y \rightarrow \eta$ on $J$. For brevity, the process of summability so defined will be called a $K$-method. 
Theorem. For the regularity of a $K$-method in the class ( $\mathfrak{B})$ it is necessary and sufficient that (a) $\int_{0}^{\xi} K(x, y) d x$ exist on $J$ and tend to 1 as $y \rightarrow \eta$ on $J$; (b) $\int_{H} K(x, y) d x \rightarrow 0$ as $y \rightarrow \eta$ on $J$, for every measurable set $H$ on I not having $\xi$ as a limit point; (c) $\int_{0}^{\xi}|K(x, y)| d x$ exist on $J$, and that constants $M$ and $Y$ exist such that this integral is bounded by $M$ for $Y<y<\eta$.

Proof. Necessity. Since the function $g(x) \equiv 1$ on $I$ belongs to $\mathfrak{B}$ with $L_{g}=1$, the necessity of (a) is apparent. Moreover, if $h(x)$ is the characteristic function of any set $H$ of the type described in (b), then $h(x) \subset(\mathfrak{B})$ with $L_{h}=0$; thus (b) is necessary. To establish (c) we observe first that the existence of $\int_{0}^{\xi} K(x, y) d x$ implies that $\int_{0}^{X}\left|K_{1}(x, y)\right| d x, \int_{0}^{X}\left|K_{2}(x, y)\right| d x$ both exist, from which the existence of $\int_{0}^{X}|K(x, y)| d x$ follows for $y$ on $J$ and $0<X<\xi$. Suppose now, contrary to the first part of (c), that for some value of $y$, say $y_{0}$, we have $\int_{0}^{\xi}\left|K\left(x, y_{0}\right)\right| d x$ $\equiv \lim _{X \rightarrow \xi} \int_{0}^{X}\left|K\left(x, y_{0}\right)\right| d x=\infty$. Then there exists on $I$ a sequence $\left\{x_{j}\right\}$ tending to $\xi$, such that

$$
\int_{x_{j-1}}^{x_{j}}\left|K\left(x, y_{0}\right)\right| d x>j, \quad\left(j=1,2,3, \cdots ; x_{0}=0\right) .
$$

If we define $k(x)$ for $x_{j-1} \leqq x<x_{i},(j=1,2,3, \cdots)$, as zero if $K\left(x, y_{0}\right)=0$, and as $\left[K_{1}\left(x, y_{0}\right)-i K_{2}\left(x, y_{0}\right)\right] /\left(j\left|K\left(x, y_{0}\right)\right|\right)$ if $K\left(x, y_{0}\right) \neq 0$, then $k(x) \subset(\mathfrak{B})$, whereas, for $n=1,2,3, \cdots$,

$$
\int_{0}^{x_{n}} K\left(x, y_{0}\right) k(x) d x=\sum_{j=1}^{n} \frac{1}{j} \int_{x_{j-1}}^{x_{j}}\left|K\left(x, y_{0}\right)\right| d x>n,
$$

so that $U_{y_{0}}(k)$ does not exist. This contradiction proves the first part of (c). To establish the second part we first define ess.sup. $0 \leqq x<\xi|f(x)|$ as the greatest lower bound of all numbers $C$ for which $|f(x)| \leqq C$ almost everywhere. Then by introducing the functional $\|f\| \equiv$ ess.sup. $0 \leqq x<\xi|f(x)|$ as the norm, one may verify that $(\mathfrak{B})$ becomes a Banach space and that $U_{y}(f)$, for each fixed $y$ on $J$, is a linear operation in this space. Now choose $X$ arbitrarily, $0<X<\xi$, and define $f_{y}(x)$ for $0 \leqq x \leqq X$ as 1 if $K(x, y)=0$, as $\left[K_{1}(x, y)-i K_{2}(x, y)\right] /|K(x, y)|$ if $K(x, y) \neq 0$, and for $X<x<\xi$ as zero. For each value of $y$ we have $f_{y}(x) \subset(\mathfrak{B})$ with $\left\|f_{y}\right\|=1$. Hence we obtain $U_{y}\left(f_{y}\right)=\int_{0}^{X}|K(x, y)| d x$ $\leqq\left\|U_{y}\right\| \cdot\left\|f_{y}\right\|=\left\|U_{y}\right\|$, from which it follows that 


$$
\int_{0}^{\xi}|K(x, y)| d x \leqq\left\|U_{y}\right\| \text { for } y \text { on } J,
$$

since $X$ was arbitrary. Let $s \equiv\left\{y_{n}\right\}$ be any sequence on $J$ tending to $\eta$. By the assumed regularity, the sequence $\left\{U_{y_{n}}(f)\right\}$ converges for every $f \subset(\mathfrak{B})$, and hence by a theorem of Banach* there exists a number $N$, depending on $s$, such that $\left\|U_{y_{n}}\right\| \leqq N$ for $n=1,2,3, \cdots$. From this fact we infer the existence of numbers $M$ and $Y$ such that $\left\|U_{y}\right\| \leqq M$ for $Y<y<\eta$, which, by virtue of (1), completes the proof of necessity.

Sufficiency. Assuming that $K(x, y)$ satisfies (a), (b), and (c), it is clear first of all that $U_{y}(f)$ exists on $J$ for every $f \subset(\mathfrak{B})$. Now choose $f(x) \subset(\mathfrak{B})$ arbitrarily and set $f(x) \equiv L_{f}+r(x)$, where, accordingly, $r(x) \subset(\mathfrak{B})$ with $L_{r}=0$. Then $U_{y}(f)=U_{y}\left(L_{f}\right)+U_{y}(r)$ and since by condition (a), $U_{y}\left(L_{f}\right) \rightarrow L_{f}$ as $y \rightarrow \eta$ on $J$, it remains only to show that $U_{y}(r) \rightarrow 0$. To this end let $\epsilon>0$ be given; then a number $x_{0}(\epsilon)$ exists, $0<x_{0}<\xi$, such that

$$
|r(x)| \leqq(2)^{1 / 2} \epsilon /(12 M) \text { for } x_{0}<x<\xi .
$$

Furthermore, if $r(x) \equiv r_{1}(x)+i r_{2}(x)$, there exist functions $R_{j}(x)$ of the form $R_{j}(x) \equiv \sum_{n=1}^{m} a_{n j} h_{n}(x)$, where the $a_{n j}$ are real numbers and the $h_{n}(x)$ are the characteristic functions of certain measurable sets $H_{n}$ on the interval $\left(0, x_{0}\right)$, such that

(3) $\left|r_{j}(x)-R_{j}(x)\right| \leqq(2)^{1 / 2} \epsilon /(12 M)$ almost everywhere on $\left(0, x_{0}\right)$, when $j=1$ or 2 . Setting $R(x) \equiv R_{1}(x)+i R_{2}(x)$, we may write

$$
\begin{aligned}
U_{y}(r)= & \sum_{n=1}^{m}\left(a_{n 1}+i a_{n 2}\right) \int_{H_{n}} K(x, y) d x \\
& +\int_{0}^{x_{0}} K(x, y)[r(x)-R(x)] d x+\int_{x_{0}}^{\xi} K(x, y) r(x) d x .
\end{aligned}
$$

By condition (b) a number $Y_{0}(\epsilon)$ exists, $Y \leqq Y_{0}<\eta$, such that the first term on the right does not exceed $\epsilon / 3$ in absolute value for $Y_{0}<y<\eta$. Finally, making use of (2), (3), and condition (c), one readily shows that the remaining integrals are each numerically $\leqq \epsilon / 3$ for $Y_{0}<y<\eta$. Hence $\left|U_{y}(r)\right| \leqq \epsilon$ for $Y_{0}<y<\eta$, which completes the proof of the theorem.

Brown University

\footnotetext{
* Banach, Théorie des Opérations Linéaires, p. 80, Théorème 5.
} 